\title{
Determination of the bulk scattering parameters of diffusing materials
}

\author{
Sven Leyre, ${ }^{1,2,3, *}$ Frédéric B. Leloup, ${ }^{1,4}$ Jan Audenaert, ${ }^{1,2}$ Guy Durinck, ${ }^{1,2}$ \\ Johan Hofkens, ${ }^{5}$ Geert Deconinck, ${ }^{2}$ and Peter Hanselaer ${ }^{1,2}$ \\ 'Light and Lighting Laboratory, Catholic University College Ghent, Gebroeders Desmetstraat 1, Ghent 9000, Belgium \\ ${ }^{2}$ ESAT/ELECTA, KU Leuven, Kasteelpark Arenberg 10, bus 2445, Leuven 3001, Belgium \\ ${ }^{3}$ SIM (Flemish Strategic Initiative on Materials), SOPPOM program, Technologiepark 935, Zwijnaarde B-9052, Belgium \\ ${ }^{4}$ Computer Graphics Research Group, KU Leuven, Celestijnenlaan 200 A, Leuven 3001, Belgium \\ ${ }^{5}$ Department of Chemistry, KU Leuven, Celestijnenlaan 200 F, bus 2404, Leuven 3001, Belgium \\ ${ }^{*}$ Corresponding author: sven.leyre@kahosl.be
}

Received 25 February 2013; revised 16 May 2013; accepted 17 May 2013;

posted 20 May 2013 (Doc. ID 185920); published 12 June 2013

\begin{abstract}
Diffusors are widely used optical components having numerous applications. They are commonly used to homogenize light beams and to create particular intensity distributions. The angular scattering profile of bulk scattering diffusing materials is determined by three bulk scattering parameters that are, however, not commonly available. This hampers an accurate implementation of bulk diffusors in ray tracing simulations. In this paper, the bulk scattering parameters of a concentration series of milk diluted with water were determined with the inverse adding-doubling method. Using these values as input, the macroscopic angular scattering profile was simulated using ray tracing software. The simulation results were compared to experimental data, and a good agreement between measured and simulated data was found. The method was also proven to be successful when applied to commercial diffusors. () 2013 Optical Society of America

OCIS codes: （290.5820) Scattering measurements; (290.4210) Multiple scattering; (290.1483) BSDF, BRDF, and BTDF.

http://dx.doi.org/10.1364/AO.52.004083
\end{abstract}

\section{Introduction}

Diffusing materials are used in a lot of applications, such as luminaire design and display technology [1-3]. These materials redistribute the light through surface scattering, volume scattering, or a combination of both. Surface scattering occurs at the front or back surface of the material and is caused by geometrical irregularities. Volume or bulk scattering, on the other hand, occurs inside the material, and is due to the presence of scattering particles in a host material. In order to perform accurate optical

$1559-128 \mathrm{X} / 13 / 184083-08 \$ 15.00 / 0$

(C) 2013 Optical Society of America simulations, diffusing materials need to be adequately modeled.

Surface scattering is described using the bidirectional scatter distribution function (BSDF), which can be subdivided into the bidirectional reflectance distribution function (BRDF) and the bidirectional transmittance distribution function (BTDF), respectively, $[\underline{4}, \underline{5}]$. These functions are defined as the ratio of the surface radiance of the sample in a given viewing direction to the irradiance from a particular incident direction. Both the incident and viewing angles are defined by the spherical coordinates toward the sample normal, $\left(\theta_{i}, \varphi_{i}\right)$ and $\left(\theta_{s}, \varphi_{s}\right)$, respectively. The BRDF and BTDF values can be determined for each wavelength $\lambda$. 
Bulk scattering is modeled by the use of three bulk scattering parameters: the absorption coefficient $\mu_{a}$, the scattering coefficient $\mu_{s}$, and the phase function $p(\theta)$. The absorption and scattering coefficients define the probability of a photon being absorbed and scattered after travelling a unit path length through the material, and are usually expressed in $\mathrm{mm}^{-1}$. The phase function determines the probability of a photon being scattered in a given direction after a single scatter event [6].

For ray tracing simulations of, e.g., luminaires or displays, the bulk diffusor is an essential optical component, since it redirects the light both angularly and spatially. In order to model the component accurately, the bulk scattering parameters should be used as input in the ray tracing software. However, for commercial diffusors, the values are usually not available.

The determination of the bulk scattering parameters of materials has been the subject of investigation in numerous application fields. Among others, these include the medical field (to study tissue) [7-9], astronomy (to model light propagation through the galaxy or the atmosphere) [10], and the biological field (e.g., to examine food samples) [11]. Two categories of methods are commonly used: the forward method and the inverse method. In the forward method, basic physical properties (such as the refractive index of both the host material and the particle, the particle size, the size distribution function, etc.) are used to infer the bulk scattering parameters. A commonly employed forward method is the Lorenz-Mie theory, which can be used for spherical particles [6]. In the inverse method, measured macroscopic quantities, such as the reflectance, transmittance, and spatial reflectance profile of the given sample are taken as input to determine its bulk scattering parameters. The inverse method has the advantage that the basic physical properties of the scattering particles and host material need not be known. Examples of inverse methods are inverse adding-doubling (IAD) [12], inverse Monte Carlo (IMC) [13], and the use of the diffusion approximation [14].

In this paper, the IAD method was selected for the determination of the bulk scattering parameters of two types of material. The IAD method is based on the adding-doubling $(\mathrm{AD})$ method, which was first described by Stokes in 1860 [15]. Stokes developed the $\mathrm{AD}$ method to calculate the reflectance and the transmittance through a pile of plates. In the IAD method, the bulk scattering parameters are determined via an iterative procedure, starting from the reflectance, transmittance, and regular transmittance. The reflectance and transmittance are the sums of the diffuse and regular reflectance and transmittance. The IAD method has the advantage that it is fast and that the energy balance of the diffusing material will be correct, since the method seeks after scattering parameters which match the measured reflectance and transmittance. Moreover, the method does not require information on the basic physical properties (such as shape, size distribution, and refractive index) of the scattering particles, which is usually not available for commercial diffusing materials used in lighting and display technology.

The aim of this paper is to investigate the use of the IAD method to determine bulk scattering parameters and to examine if these parameters perform well when used as input for optical simulations of the angular scattering of bulk diffusors.

The method has been applied for two types of diffusing materials: a concentration series of milk diluted with water and two commercial plastic diffusors. The experimental angular scattering profile of the samples is compared to the predictions from ray tracing simulations.

\section{Determination of Bulk Scattering Parameters}

\section{A. Adding-Doubling}

The $\mathrm{AD}$ method has been extensively described in literature by numerous authors [15-17]. The method can be used to obtain the reflectance and transmittance of a slab of material with thickness $d$ with known bulk scattering parameters. The procedure requires three steps.

First, the angular reflection and transmission characteristics of a "single scatter layer" are determined. The single scatter layer is a thin layer with identical scattering properties to the sample in question, and a thickness such that a photon will be scattered only once while travelling through the layer. The reflection and transmission characteristics of such a layer can be found by solving the radiative transfer equation, given in Eq. (1):

$$
\begin{aligned}
\mathbf{s .} \nabla L(\mathbf{r}, \mathbf{s})= & -\left(\mu_{a}+\mu_{s}\right) L(\mathbf{r}, \mathbf{s}) \\
& +\mu_{s} \int_{4 \pi} p\left(\mathbf{s}, \mathbf{s}^{\prime}\right) L\left(\mathbf{r}, \mathbf{s}^{\prime}\right) \mathrm{d} \Omega^{\prime} .
\end{aligned}
$$

Herein $\mathbf{s}$ is the direction vector of the incident direction, $\mathbf{s}^{\prime}$ the direction vector of the scattered direction, and $\mathbf{r}$ the position vector. $L(\mathbf{r}, \mathbf{s})$ is the (spectral) radiance or luminance at position $\mathbf{r}$ and in direction $\mathbf{s}$, and $\Omega^{\prime}$ is the solid angle in which the (spectral) radiance or luminance is considered. $p\left(\mathbf{s}, \mathbf{s}^{\prime}\right)$ is the phase function, which gives the probability that a photon with incident direction $\mathbf{s}$ will be scattered into direction $\mathbf{s}^{\prime}$ [16].

Second, the thickness of the layer is doubled, and the reflection and transmission characteristics of the double layer are calculated by combining the reflection and transmission characteristics of the single scatter layers [15-17]. The thickness of this new layer is then again doubled, and the new reflection and transmission characteristics are again calculated, until the thickness of the sample in question has been reached.

The third and last step is to take the Fresnel reflection at the interfaces into account by adding an additional boundary layer [12]. 
In this way, the reflectance $R$ and transmittance $T$ of a material with known volume scattering properties can be calculated. Finally, the regular transmittance $\left(T_{\text {reg }}\right)$ of a sample with thickness $d$ can be calculated from Eq. ( $\underline{2})$ :

$$
T_{\text {reg }}=\frac{(1-r)^{2} e^{-\tau}}{1-r^{2} e^{-2 . \tau}} .
$$

$T_{\text {reg }}$ is defined as the fraction of the incident flux transmitted through the sample without scattering. In Eq. (2), $\tau$ represents the optical thickness, defined as $\tau=\left(\mu_{a}+\mu_{s}\right) \cdot d$, and $r$ is the Fresnel power reflection coefficient for unpolarized light.

Note that light travelling in the direction of the regular transmission (regular direction) consists of the actual regular transmittance described by $T_{\text {reg }}$ and a contribution of scattered light in the regular direction.

\section{B. Inverse Adding-Doubling}

An iterative procedure is adopted in the IAD method. First, a guess is made for the bulk scattering parameters $\mu_{a}, \mu_{s}$, and $p(\theta)$. Next, the reflectance, transmittance, and regular transmittance are calculated with the $\mathrm{AD}$ method. If there is no match between the calculated and the measured quantities, the initial bulk scattering parameters are adjusted until a match is obtained [12].

Theoretically, any phase function $p(\theta)$ can be used in the $\mathrm{AD}$ method. However, too many free parameters would decelerate the convergence of the IAD method. To avoid this problem, a fixed phase function is commonly used-the Henyey-Greenstein phase function $p_{\mathrm{HG}}(\theta)$, given by Eq. (3) [18]:

$$
p_{H G}(\theta)=\frac{1}{4 \pi} \frac{1-g^{2}}{\left(1+g^{2}-2 g \cos (\theta)\right)^{3 / 2}} .
$$

The only variable in the phase function is the anisotropy factor $g$, which is the average cosine of the scattering angle $\theta$, and which can take values from -1 to 1 . If $g$ equals 0 , scattering occurs isotropically; if $g$ is higher or lower than 0 , scattering occurs preferentially in the forward or backward direction, respectively.

Since this phase function allows only one parameter to vary, the number of free parameters for the IAD procedure is limited to three: $\mu_{a}, \mu_{s}$, and $g$.

If it is not possible to measure the regular transmittance, either due to equipment limitations or because it is too small to be measured accurately, there are only two input parameters, and only two output parameters can be found. Indeed, if the scattering is strong and dominant over the absorption, the radiative transfer can be classified to be in the diffusion regime. In this case, the reduced scattering coefficient $\mu_{s}^{\prime}$ is the more relevant parameter [19]. The reduced scattering coefficient can be obtained by combining $\mu_{s}$ and $g$ according to the similarity relation, given by Eq. (4) [19]:

$$
\mu_{s}^{\prime}=\mu_{s}(1-g)
$$

Any combination of $\mu_{s}$ and $g$ satisfying Eq. (4) will result in an identical reflectance and transmittance, as long as the regular transmittance is significantly small (typically $<0.005$ ).

\section{Experiments}

In order to investigate the usefulness of the IAD method to determine bulk scattering parameters, two types of diffusing materials were investigated: a series of diluted milk in a glass cuvette and two commercial plastic diffusors. The commercial plastic diffusors were purchased from Evonik, materials WH02 and WN297. The milk series consisted of ultrahigh-temperature processed semi-skimmed cow milk, diluted with water. Three concentrations were chosen $(10,15$, and 20 vol. \%), high enough to minimize light propagation to the edge of the cuvette and low enough to obtain a significant regular transmittance. The glass cuvette itself exhibited negligible absorption and scattering, and the Fresnel reflections from the air-glass and the glass-milk interfaces were taken into account in the IAD calculations by adding extra boundary layers [11,12].

Performing correct reflectance and transmittance measurements of translucent samples by use of standard spectrophotometers is not evident, due to the spatial redistribution of the light. The results can be strongly dependent on the illumination and detection geometry.

A schematic representation of the setup used in this study is given in Fig. 1 [5]. The setup provides information about the angular distribution of the scattered light, which can be compared with ray tracing simulations. The setup consists of two major parts: the illumination part and the detection part. The illumination part comprises a Xe light source and imaging optics, which create a parallel beam of light incident on the sample. A set of neutraldensity (ND) filters is introduced into the light beam to increase the system's dynamic range. The detection part consists of a detector head, which is connected to a spectrometer with a CCD detector via an optical fiber. The detector has a sufficiently large field of view in order to be sure that all the scattered

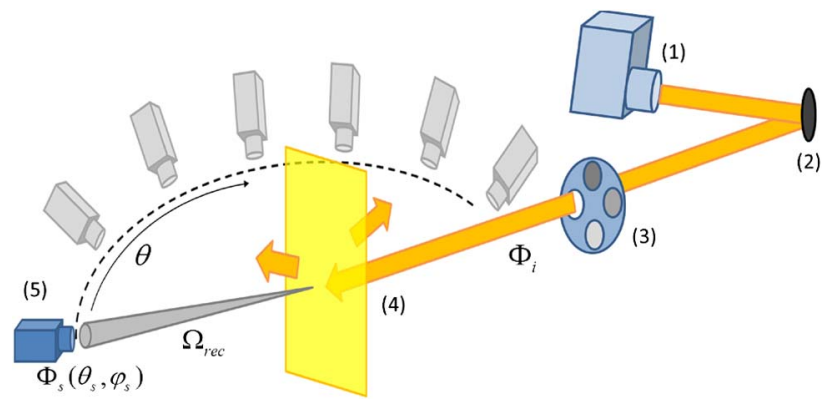

Fig. 1. Schematic representation of the BSDF setup: (1) Xe source, (2) collimating mirror, (3) ND filter wheel, (4) sample, and (5) detector head. 
light will be captured. The entire unit is mounted on a detector bench, which can be rotated into any position around a sample holder by the use of two automated stepper motors. As such, the setup allows for the measurement of the scattered intensities in both reflection and transmission mode. A more elaborate description of the setup can be found in [5].

All measurements were performed under normal incident light to the sample. The scattered radiant intensities were measured in one plane in increments of 10 degrees. Since the scattering particles of both materials used in the study have no preferential orientation, scattering is assumed to be symmetric with respect to the azimuth angle. This assumption is also a prerequisite for the application of the IAD procedure. The reflectance $R$ and transmittance $T$ can be calculated from the scattered spectral radiant intensities $I_{s}(\theta)$ according to Eqs. (5) and (6):

$$
\begin{gathered}
T=\frac{2 \pi}{\Phi_{i}} \int_{0}^{\pi / 2} I_{s}(\theta) \sin (\theta) \mathrm{d} \theta+T_{\mathrm{reg}}, \\
R=\frac{2 \pi}{\Phi_{i}} \int_{\pi / 2}^{\pi} I_{s}(\theta) \sin (\theta) \mathrm{d} \theta+R_{\mathrm{reg}} .
\end{gathered}
$$

Herein $\Phi_{i}$ stands for the incident spectral radiant flux, which can be measured by removing the sample from the sample holder and measuring the entire incident beam. This is very easy because the beam diameter is smaller than the detector aperture. $R_{\text {reg }}$ represents the regular reflectance.

The regular transmittance $T_{\text {reg, }}$, which is defined as the fraction of the total incident light transmitted through the material without being scattered, can be measured by performing a measurement at $\theta_{s}=0^{\circ}$. However, as the detector solid angle $\Omega_{\text {rec }}$ is not infinitesimally small, this measurement also includes a fraction of light scattered in the regular direction. In some cases, this contribution can be negligibly small in comparison with the actual regular transmittance. However, if the regular transmittance is low, this effect should be accounted for. The fraction of scattered light in the regular direction can be estimated by extrapolating the values of $I_{s}$ close to the normal viewing angle. For a perfect Lambertian reflecting or transmitting diffuser, the spectral radiant intensity divided by the cosine of the viewing angle should remain constant across the viewing angle $\theta_{s}$. Since the diffusing materials under testing exhibit a Lambertian or near-Lambertian angular scattering profile, the extrapolation has been based on the spectral radiant intensities divided by the cosine of the scatter angle, given by Eq. (7):

$$
I_{s}\left(0^{\circ}\right)=\frac{I_{s}\left(\theta_{1}\right)}{\cos \left(\theta_{1}\right)}+\left(\frac{I_{s}\left(\theta_{1}\right)}{\cos \left(\theta_{1}\right)}-\frac{I_{s}\left(\theta_{2}\right)}{\cos \left(\theta_{2}\right)}\right) \frac{\theta_{1}}{\left(\theta_{1}-\theta_{2}\right)} .
$$

The angles $\theta_{1}$ and $\theta_{2}$ are the smallest scatter directions next to the regular direction (in this case $\theta_{1}=10^{\circ}$ and $\left.\theta_{2}=20^{\circ}\right)$. The regular transmittance $T_{\text {reg }}$ can be calculated from Eq. ( $\left.\underline{8}\right)$ :

$$
T_{\mathrm{reg}}=\frac{\Phi_{s}\left(0^{\circ}\right)-I_{s}\left(0^{\circ}\right) \Omega_{\mathrm{rec}}}{\Phi_{i}}
$$

In Eq. (8), $\Phi_{s}\left(0^{\circ}\right)$ is the spectral radiant flux measured with the detector head in the regular direction, $\Phi_{i}$ is the incident flux on the sample, and $\Omega_{\mathrm{rec}}$ is the solid angle from the sample to the detector aperture. $I_{s}\left(0^{\circ}\right)$ is calculated with Eq. (7). The regular reflectance $R_{\text {reg }}$ can be calculated in a similar way. The measurement for $I_{s}\left(180^{\circ}\right)$ was taken at scatter angle $176^{\circ}$, with the incident angle at $2^{\circ}$, since an actual measurement at $180^{\circ}$ with incident angle $0^{\circ}$ is not possible.

The results of the measurements performed on both types of diffusing materials are presented in Table 1. All data were obtained at three wavelengths: $480 \mathrm{~nm}$ (blue), $550 \mathrm{~nm}$ (green), and $630 \mathrm{~nm}$ (red). The thickness of the samples is given in parentheses. For the diluted milk series, the thickness refers to the internal thickness of the cuvettes. The regular transmittance is corrected using Eqs. (7) and (8). For the commercial diffusors, the regular transmittance could not be accurately determined (smaller than $1 \%$ of the scattered flux in the forward direction), and it is therefore not included in the measurement

\begin{tabular}{|c|c|c|c|}
\hline Sample & $R$ & $T$ & $T_{\text {reg }}$ \\
\hline \multicolumn{4}{|l|}{ Wavelength: $480 \mathrm{~nm}$} \\
\hline Diffusor WH02 (3 mm) & 0.51 & 0.48 & - \\
\hline Diffusor WN297 (3 mm) & 0.94 & 0.03 & - \\
\hline Milk 10 vol. \% (1.1 mm) & 0.23 & 0.74 & 0.131 \\
\hline Milk 15 vol. \% (1.1 mm) & 0.31 & 0.66 & 0.027 \\
\hline Milk 20 vol. $\%(1.1 \mathrm{~mm})$ & 0.37 & 0.61 & 0.0092 \\
\hline Milk 10 vol. \% (2 mm) & 0.30 & 0.65 & 0.032 \\
\hline Milk 15 vol. \% (2 mm) & 0.41 & 0.52 & 0.0016 \\
\hline Milk 20 vol. \% (2 mm) & 0.45 & 0.48 & 0.0003 \\
\hline \multicolumn{4}{|l|}{ Wavelength: $550 \mathrm{~nm}$} \\
\hline Diffusor WH02 (3 mm) & 0.49 & 0.49 & - \\
\hline Diffusor WN297 (3 mm) & 0.93 & 0.03 & - \\
\hline Milk 10 vol. $\%(1.1 \mathrm{~mm})$ & 0.20 & 0.77 & 0.226 \\
\hline Milk 15 vol. \% (1.1 mm) & 0.28 & 0.70 & 0.072 \\
\hline Milk 20 vol. $\%(1.1 \mathrm{~mm})$ & 0.34 & 0.65 & 0.033 \\
\hline Milk 10 vol. \% (2 mm) & 0.27 & 0.69 & 0.081 \\
\hline Milk 15 vol. \% (2 mm) & 0.37 & 0.56 & 0.0088 \\
\hline Milk 20 vol. $\%$ (2 mm) & 0.42 & 0.53 & 0.0024 \\
\hline \multicolumn{4}{|l|}{ Wavelength: $630 \mathrm{~nm}$} \\
\hline Diffusor WH02 (3 mm) & 0.47 & 0.50 & - \\
\hline Diffusor WN297 (3 mm) & 0.91 & 0.03 & - \\
\hline Milk 10 vol. \% (1.1 mm) & 0.17 & 0.79 & 0.331 \\
\hline Milk 15 vol. \% (1.1 mm) & 0.24 & 0.73 & 0.145 \\
\hline Milk 20 vol. \% (1.1 mm) & 0.30 & 0.68 & 0.081 \\
\hline Milk 10 vol. \% (2 mm) & 0.23 & 0.72 & 0.158 \\
\hline Milk 15 vol. \% (2 mm) & 0.33 & 0.60 & 0.031 \\
\hline Milk 20 vol. \% (2 mm) & 0.38 & 0.56 & 0.012 \\
\hline
\end{tabular}
results.

Table 1. Measured Reflectance, Transmittance, and Regular Transmittance of the Diffusing Materials 
The data in Table 1 show that for all samples the reflectance is slightly larger at short wavelengths, while the transmittance increases with longer wavelengths. This indicates that the scattering is stronger at shorter wavelengths. The effect is more prominently present for the diluted milk series compared to the commercial diffusors.

From the measurement results in Table 1, the optical scattering parameters can now be determined. Since the regular transmittance was measured to be negligible for the two commercial diffusors, only two scattering parameters are calculated $\left(\mu_{s}^{\prime}\right.$ and $\left.\mu_{\alpha}\right)$. For the milk series, on the contrary, all three scattering parameters $\left(\mu_{a}, \mu_{s}\right.$, and $\left.g\right)$ are determined.

In Table $\underline{2}$, the final bulk scattering parameters are presented, together with the values for the reflectance, transmittance, and the regular transmittance as obtained with the IAD procedure. The relative deviation from the experimental values is indicated between brackets. For all results, the deviation is smaller than $5 \%$.

For the diluted milk series, the values represented in Table 2 show an expected course, the scattering coefficient $\mu_{s}$ of all three concentrations is very similar for both cuvette thicknesses at all wavelengths. This can be expected, since the scattering coefficient is independent of the thickness of the sample. The scattering coefficient decreases with increasing wavelength, which can be expected from small scattering fat molecules. For the absorption coefficient $\mu_{a}$, the agreement between the parameters determined for the different thicknesses is less pronounced. This is due to the fact that the absorption coefficient is several magnitudes smaller than the scattering coefficient, which limits the accuracy that can be reached. The wavelength dependency of the absorption coefficient is negligible. The anisotropy factor $g$ is very similar for all six samples and decreases with increasing wavelength. The scattering occurs preferentially in the forward direction for all wavelengths $(g>0)$.

For the commercial diffusors, the highest reduced scattering coefficient is obtained for the strongest diffusing sample. Both diffusors exhibit very low absorption. The diffusors also show a decreasing reduced scattering coefficient at longer wavelengths, but the effect is less pronounced than with the diluted milk series.

\section{Verification of Angular Scattering}

The values of the bulk scattering parameters as determined in the previous section were introduced in ray tracing software to simulate the angular scattering pattern of the bulk diffusor. The commercial ray tracing software TracePro was used. The software uses traditional Monte Carlo ray tracing code. Bulk scattering is modeled with three bulk scattering parameters: the absorption coefficient, the scattering coefficient, and the phase function. The ray tracer allows the use of the Henyey-Greenstein, the Gegenbauer, or a user defined phase function.

The software allows the creation of polar intensity distribution maps, which can be compared to the measured data. In the ray tracing software, the

Table 2. Bulk Scattering Properties of the Diffusing Materials Determined with the IAD Method

\begin{tabular}{|c|c|c|c|c|c|c|c|}
\hline Sample & $\mu_{a}\left[\mathrm{~mm}^{-1}\right]$ & $\mu_{s}\left[\mathrm{~mm}^{-1}\right]$ & $g$ & $\mu_{s}^{\prime}\left[\mathrm{mm}^{-1}\right]$ & $R(\Delta R)$ & $T(\Delta T)$ & $T_{\text {reg }}\left(\Delta T_{\text {reg }}\right)$ \\
\hline \multicolumn{8}{|l|}{ Wavelength: $480 \mathrm{~nm}$} \\
\hline Diffusor WH02 (3 mm) & 0.0012 & - & - & 0.696 & $0.51(0.0 \%)$ & $0.48(0.2 \%)$ & - \\
\hline Diffusor WN297 (3 mm) & 0.0025 & - & - & 24.876 & $0.94(0.0 \%)$ & $0.03(0.5 \%)$ & - \\
\hline Milk 10 vol. \% (1.1 mm) & 0.0071 & 1.757 & 0.78 & 0.387 & $0.24(3.7 \%)$ & $0.74(0.3 \%)$ & $0.131(0.0 \%)$ \\
\hline Milk 15 vol. \% (1.1 mm) & 0.0010 & 3.204 & 0.785 & 0.689 & $0.32(3.7 \%)$ & $0.64(1.9 \%)$ & $0.027(0.0 \%)$ \\
\hline Milk 20 vol. $\%(1.1 \mathrm{~mm})$ & 0.0042 & 4.178 & 0.785 & 0.898 & $0.38(0.8 \%)$ & $0.61(0.3 \%)$ & $0.0092(0.0 \%)$ \\
\hline Milk 10 vol. \% (2 mm) & 0.0067 & 1.676 & 0.785 & 0.360 & $0.31(3.1 \%)$ & $0.65(0.9 \%)$ & $0.032(0.0 \%)$ \\
\hline Milk 15 vol. $\%$ (2 mm) & 0.0095 & 3.165 & 0.78 & 0.696 & $0.41(1.7 \%)$ & $0.52(0.1 \%)$ & $0.0016(0.0 \%)$ \\
\hline Milk 20 vol. $\%$ (2 mm) & 0.0081 & 4.045 & 0.785 & 0.870 & $0.46(0.8 \%)$ & $0.49(0.6 \%)$ & $0.0003(0.0 \%)$ \\
\hline \multicolumn{8}{|l|}{ Wavelength: $550 \mathrm{~nm}$} \\
\hline Diffusor WH02 (3 mm) & 0.0014 & - & - & 0.632 & $0.49(0.2 \%)$ & $0.49(0.3 \%)$ & - \\
\hline Diffusor WN297 (3 mm) & 0.0027 & - & - & 23.264 & $0.93(0.0 \%)$ & $0.03(0.0 \%)$ & - \\
\hline Milk 10 vol. $\%(1.1 \mathrm{~mm})$ & 0.0083 & 1.265 & 0.755 & 0.310 & $0.21(4.4 \%)$ & $0.77(0.6 \%)$ & $0.226(0.0 \%)$ \\
\hline Milk 15 vol. $\%(1.1 \mathrm{~mm})$ & 0.0081 & 2.308 & 0.760 & 0.554 & $0.29(4.7 \%)$ & $0.68(1.8 \%)$ & $0.072(0.0 \%)$ \\
\hline Milk 20 vol. $\%(1.1 \mathrm{~mm})$ & 0.0055 & 3.029 & 0.750 & 0.757 & $0.34(1.9 \%)$ & $0.64(2.1 \%)$ & $0.033(0.0 \%)$ \\
\hline Milk 10 vol. \% (2 mm) & 0.0087 & 1.204 & 0.760 & 0.289 & $0.27(2.1 \%)$ & $0.68(2.2 \%)$ & $0.081(0.0 \%)$ \\
\hline Milk 15 vol. \% (2 mm) & 0.0086 & 2.314 & 0.760 & 0.555 & $0.38(1.4 \%)$ & $0.57(0.2 \%)$ & $0.0088(0.0 \%)$ \\
\hline Milk 20 vol. \% (2 mm) & 0.0074 & 2.964 & 0.760 & 0.711 & $0.42(0.5 \%)$ & $0.53(0.7 \%)$ & $0.0024(0.0 \%)$ \\
\hline \multicolumn{8}{|l|}{ Wavelength: $630 \mathrm{~nm}$} \\
\hline Diffusor WH02 (3 mm) & 0.0022 & - & - & 0.561 & $0.47(0.2 \%)$ & $0.50(0.2 \%)$ & - \\
\hline Diffusor WN297 (3 mm) & 0.0047 & - & - & 19.196 & $0.91(0.0 \%)$ & $0.03(0.6 \%)$ & - \\
\hline Milk 10 vol. $\%(1.1 \mathrm{~mm})$ & 0.0111 & 0.915 & 0.735 & 0.242 & $0.18(4.2 \%)$ & $0.79(0.5 \%)$ & $0.331(0.0 \%)$ \\
\hline Milk 15 vol. $\%(1.1 \mathrm{~mm})$ & 0.0117 & 1.663 & 0.74 & 0.432 & $0.25(3.4 \%)$ & $0.71(1.8 \%)$ & $0.145(0.0 \%)$ \\
\hline Milk 20 vol. $\%(1.1 \mathrm{~mm})$ & 0.0066 & 2.200 & 0.735 & 0.583 & $0.30(1.3 \%)$ & $0.68(0.8 \%)$ & $0.081(0.0 \%)$ \\
\hline Milk 10 vol. \% (2 mm) & 0.0097 & 0.868 & 0.74 & 0.226 & $0.23(2.4 \%)$ & $0.71(1.7 \%)$ & $0.158(0.0 \%)$ \\
\hline Milk 15 vol. \% (2 mm) & 0.0118 & 1.676 & 0.74 & 0.436 & $0.33(0.3 \%)$ & $0.60(0.2 \%)$ & $0.031(0.0 \%)$ \\
\hline Milk 20 vol. \% (2 mm) & 0.0087 & 2.158 & 0.74 & 0.561 & $0.38(0.4 \%)$ & $0.56(0.1 \%)$ & $0.012(0.0 \%)$ \\
\hline
\end{tabular}



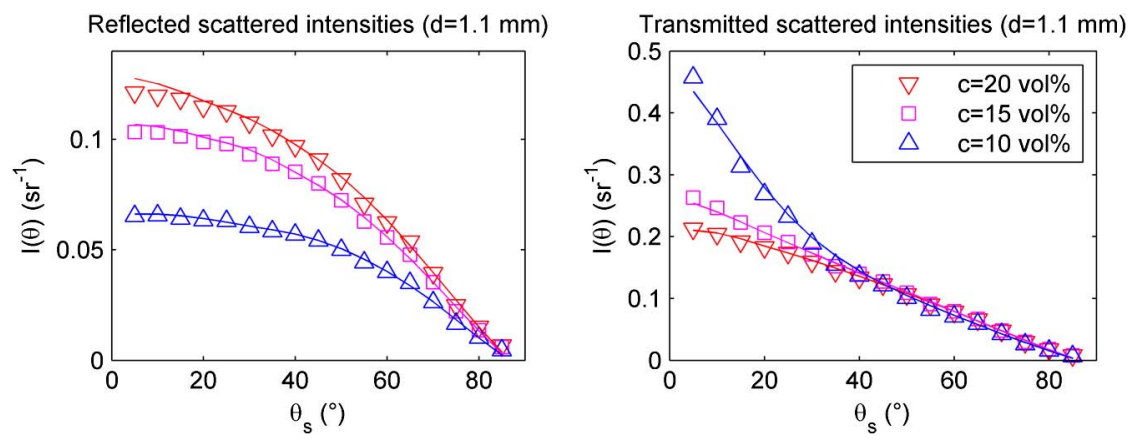

Fig. 2. Measured (marks) and simulated (curves) scattered intensities in reflection (left) and transmission (right) of a diluted milk series in a cuvette with thickness $1.1 \mathrm{~mm}$ at $550 \mathrm{~nm}$.
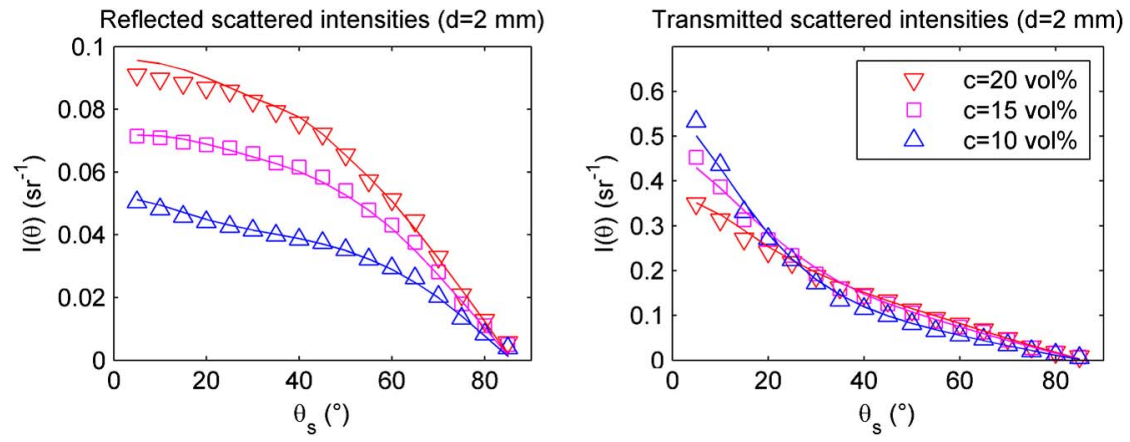

Fig. 3. Measured (marks) and simulated (curves) scattered intensities in reflection (left) and transmission (right) of a diluted milk series in a cuvette with thickness $2 \mathrm{~mm}$ at $550 \mathrm{~nm}$.

default solid angle $\Omega_{\text {sim }}$ used to calculate the scattered intensities is $0.004873 \mathrm{sr}$. All simulations were performed at $480 \mathrm{~nm}$ (blue), $550 \mathrm{~nm}$ (green), and $630 \mathrm{~nm}$ (red). The Henyey-Greenstein phase function was used for all samples.

For the diluted milk series, the scattered intensity distribution at normal incidence obtained from the simulation and from the measured data is presented in Figs. 2 and 3 for the series with the 1.1 and $2 \mathrm{~mm}$ cuvettes, respectively, at $550 \mathrm{~nm}$. The scattered intensities in the regular direction are omitted because they are masked by the regular transmission and the regular reflection. From Figs. 2 and 3 , a good agreement between the scattered intensity patterns from experiments and simulations can be observed. Similar results were obtained at 480 and $630 \mathrm{~nm}$.

To quantify the agreement between simulated and measured angular scattering, the normalized cross correlation (NCC) for the scattered intensity pattern was calculated from Eq. (ㅁ) $[\underline{20}, \underline{21}]$ :

$$
\mathrm{NCC}=\frac{\sum_{\theta=0^{\circ}}^{90^{\circ}}\left[\left(I_{m}(\theta)-\bar{I}_{m}\right)\left(I_{s}(\theta)-\bar{I}_{s}\right)\right]}{\sqrt{\sum_{\theta=0^{\circ}}^{90^{\circ}}\left[\left(I_{m}(\theta)-\bar{I}_{m}\right)^{2}\right] \sum_{\theta=0^{\circ}}^{90^{\circ}}\left[\left(I_{s}(\theta)-\bar{I}_{s}\right)^{2}\right]}} .
$$

In Eq. $\underline{9}, I_{m}(\theta)$ and $I_{s}(\theta)$ are the measured and simulated scattered intensities for each scatter angle $\theta$, respectively. $\bar{I}_{m}$ and $\bar{I}_{s}$ are the average values for the measured and simulated intensities, respectively.

The minimum NCC value for all samples is 0.9970 . The average value for all patterns is 0.9987 . For most applications, a NCC value of at least 0.99 and a maximum relative deviation of $5 \%$ are the target values in order to quantify the correspondence as very good [21]. Since both conditions are fulfilled for our data (see also Table 2), it can be concluded that the determination of bulk scattering parameters using IAD indeed allows for an accurate simulation of the macroscopic angular behavior of the diluted milk series.

Table 3. Bulk Scattering Parameters Used for the Simulations of the Two Commercial Diffusers

\begin{tabular}{clcl}
\hline \multicolumn{2}{c}{ Sample: WH02 } & \multicolumn{2}{c}{ Sample: WN297 } \\
\cline { 2 - 3 }$\mu_{s}\left[\mathrm{~mm}^{-1}\right]$ & \multicolumn{1}{c}{$g$} & $\mu_{s}\left[\mathrm{~mm}^{-1}\right]$ & $g$ \\
\hline \multirow{4}{*}{3.865} & Wavelength: & $480 \mathrm{~nm}$ \\
4.638 & 0.82 & 24.876 & 0 \\
6.957 & 0.85 & 41.459 & 0.4 \\
& 0.9 & 82.918 & 0.7 \\
3.854 & Wavelength: & $550 \mathrm{~nm}$ & \\
4.213 & 0.836 & 23.264 & 0 \\
6.320 & 0.85 & 38.773 & 0.4 \\
& 0.9 & 77.545 & 0.7 \\
3.869 & Wavelength: $630 \mathrm{~nm}$ & \\
4.315 & 0.855 & 19.196 & 0 \\
5.610 & 0.87 & 31.993 & 0.4 \\
& 0.9 & 63.986 & 0.7 \\
\hline
\end{tabular}



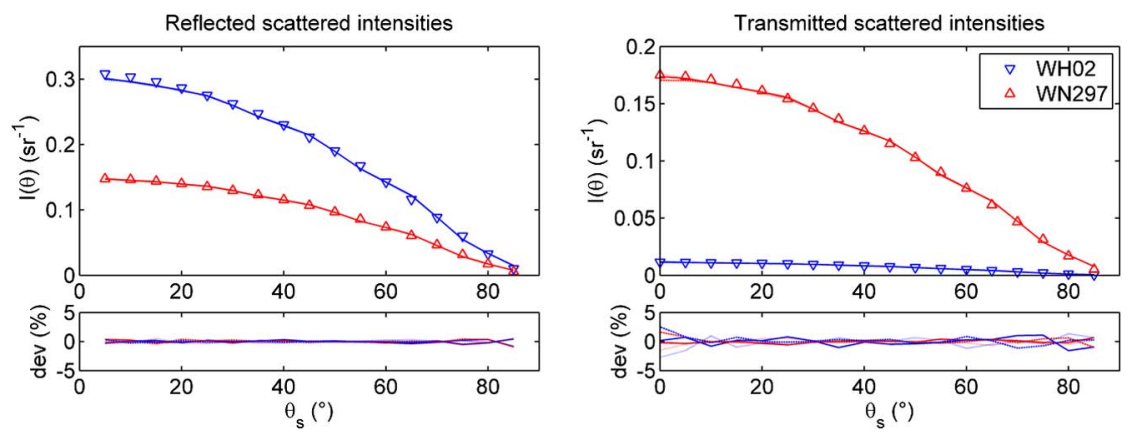

Fig. 4. Scattered intensities of the two commercial diffusors, blue for WN297 and red for WH02 at $550 \mathrm{~nm}$. The solid, dashed, and dotted curves denote the simulated scattered intensities for the three different combinations of $\mu_{s}$ and $g$ given in Table 3 . The marks are the measured values ( $\Delta$ for WH02 and $\nabla$ for WN297). The deviations between the average simulated values and the individual simulation results for each combination of $\mu_{s}$ and $g$ are shown separately below the figure.

For both commercial diffusors, only two scattering parameters, $\mu_{a}$ and $\mu_{s}^{\prime}$, could be determined. However, the three parameters $\mu_{a}, \mu_{s}$, and $g$ are required to perform the ray tracing simulation. As discussed in the introduction section, parameters $\mu_{s}$ and $g$ can be inferred from $\mu_{s}^{\prime}$ by the use of Eq. (4). In principle, an infinite number of combinations is possible. The only restriction on the choice of parameters $\mu_{s}$ and $g$ is that the regular transmittance $T_{\text {reg }}$ must remain negligible.

For both diffusors, the three combinations of $\mu_{s}$ and $g$ used in the simulations are given in Table 3 .

In Fig. $\underline{4}$, the simulation results $\left(\theta_{\mathbf{i}}=0^{\circ}\right)$ at $5 \overline{5} 0 \mathrm{~nm}$ for the two commercial diffusors are represented for three different combinations of $\mu_{s}$ and $g$, using solid, dashed, and dotted lines. It is almost impossible to distinguish the differences between the angular scattering profiles of the three combinations of $\mu_{s}$ and $g$. For both samples, all simulated values are in very good agreement with the measured scattered intensities. Similar results were obtained at 480 and $630 \mathrm{~nm}$.

The NCC values for all combinations of $\mu_{s}$ and $g$ for both samples and all wavelengths are all larger than 0.9980 , the average NCC value for all simulations being 0.9995 . The relative deviation for the reflectance and transmittance between measurements and simulations is lower than 1\% (see Table 2). The results obtained for the commercial diffusors are thus well suited for simulation of the angular scattering pattern.

\section{Conclusion}

In this paper, it has been verified that the bulk scattering parameters for bulk diffusing materials obtained with the IAD method can sufficiently predict the angular scattering pattern for two kinds of samples, without knowledge of the basic physical properties of the scattering particles (such as size distribution, shape, or refractive index). The parameters were determined at three wavelengths: $480 \mathrm{~nm}$ (blue), $550 \mathrm{~nm}$ (green), and $630 \mathrm{~nm}$ (red).

The first group of samples consists of a series of milk diluted with water, showing a significant regular transmittance. For this group of samples, all three bulk scattering parameters were determined. A good agreement between measured and simulated scattered intensity patterns was found for all samples and all wavelengths. In the future, a more elaborate study will be initiated to investigate the relationship between the bulk scattering parameters and the concentration.

The commercial samples in the second group are strongly diffusing, have a near-Lambertian scattering distribution pattern, and show no significant regular transmittance. As a consequence, only two scattering parameters $\left(\mu_{s}^{\prime}\right.$ and $\mu_{a}$ ) could be determined. It was shown that any combination of $\mu_{s}$ and $g$ can be used, as long as the value of $\mu_{s}^{\prime}$ is maintained and the regular transmittance remains negligible. All simulations resulted in the same scattered intensity distribution. It is, however, beneficial to choose $\mu_{s}$ to be as low as possible (but still high enough to have a negligible regular transmittance) to reduce computation time, since fewer scattering events imply faster ray tracing simulations.

For all simulations, a fixed Henyey-Greenstein phase function with one variable was used, instead of, e.g., the more accurate Mie phase function. From our results, it seems that this phase function can accurately describe the scattered intensity pattern for strong diffusing samples with a near-Lambertian scattering pattern, as well as samples showing only moderate scattering and appreciable regular transmission. It is known that for small diffusing particles, such as fat molecules present in milk, the Mie phase function is rather smooth. In these cases, it seems acceptable that the smooth HenyeyGreenstein phase function is able to describe the scattering process rather accurately. More research needs to be done if larger scattering particles are being used, where patterned Mie phase functions can be expected. Probably, the use of more sophisticated phase functions will then be necessary.

The authors would like to thank the SIM (Flemish Strategic Initiative for Materials) and IWT (Flemish agency for Innovation by Science and Technology) for their financial support through the SoPPoM project 
within the SIBO program. Jan Audenaert thanks the Agency for Innovation by Science and Technology in Flanders (IWT) for financial support (SB-091442).

\section{References}

1. Z. Sun, J. Chang, N. Zhao, W. Jin, and Y. Wang, "Research of diffusing plates for LCD backlights," Optik 121, 760-764 (2010).

2. J.-H. Wang, S.-Y. Lien, J.-R. Ho, T.-K. Shih, C.-F. Chen, C.-C. Chen, and W.-T. Whang, "Optical diffusers based on silicone emulsions," Opt. Mater. 32, 374-377 (2009).

3. C.-C. Sun, W.-T. Chien, I. Moreno, C.-T. Hsieh, M.-C. Lin, S.-L. Hsiao, and X.-H. Lee, "Calculating model of light transmission efficiency of diffusers attached to a lighting cavity, Opt. Express 18, 6137-6148 (2010).

4. F. E. Nicodemus, J. C. Richmond, J. J. Hsia, I. W. Ginsberg, and T. Limperis, "Geometrical considerations and nomenclature for reflectance," Final Report (National Bureau of Standards, 1977).

5. F. B. Leloup, S. Forment, P. Dutré, M. Pointer, and P. Hanselaer, "Design of an instrument for measuring the spectral bidirectional scatter distribution function," Appl. Opt. 47, 5454-5467 (2008).

6. C. F. Bohren and D. R. Huffman, Absorption and Scattering of Light by Small Particles (Wiley, 1983).

7. J. F. Beek, P. Blokland, P. Posthumus, M. Aalders, J. W. Pickering, H. J. C. M. Sterenborg, and M. J. C. van Gemert, "In vitro double-integrating-sphere optical properties of tissues between 630 and 1064 nm," Phys. Med. Biol. 42, 2255-2261 (1997).

8. J. W. Pickering, S. A. Prahl, N. van Wieringen, J. F. Beek, H. J. C. M. Sterenborg, and M. J. C. van Gemert, "Doubleintegrating-sphere system for measuring the optical properties of tissue," Appl. Opt. 32, 399-410 (1993).

9. A. Kienle, M. S. Patterson, L. Ott, and R. Steiner, "Determination of the scattering coefficient and the anisotropy factor from laser Doppler spectra of liquids including blood," Appl. Opt. 35, 3404-3412 (1996).
10. P. Minnis, K.-N. Liou, and Y. Takano, "Inference of cirrus cloud properties using satellite observed visible and infrared radiances. Part I: parameterization of radiance fields," J. Atmos. Sci. 50, 1279-1304 (1993).

11. W. Saeys, M. A. Velazco-Roa, S. N. Thennadil, H. Ramon, and B. M. Nicolaï, "Optical properties of apple skin and flesh in the wavelength range from 350 to $2200 \mathrm{~nm}$," Appl. Opt. 47, 908-919 (2008).

12. S. A. Prahl, M. J. C. van Gemert, and A. J. Welch, "Determining the optical properties of turbid media by using the adding-doubling method," Appl. Opt. 32, 559-568 (1993).

13. R. Zhang, W. Verkruysse, B. Choi, J. A. Viator, B. Jung, L. O. Svaasand, G. Aguilar, and J. S. Nelson, "Determination of human skin optical properties from spectrophotometric measurements based on optimization by genetic algorithms," J. Biomed. Opt. 10, 024030 (2005).

14. H. Karlsson, I. Fredriksson, M. Larsson, and T. Strömberg, "Inverse Monte Carlo for estimation of scattering and absorption in liquid optical phantoms," Opt. Express 20, 12233-12246 (2012).

15. G. G. Stokes, "On the intensity of the light reflected from or transmitted through a pile of plates," Proc. R. Soc. Lond. 11, $545-556$ (1860).

16. W. J. Wiscombe, "On initialization, error and flux conservation in the doubling method," J. Quant. Spectrosc. Radiat. Transfer 16, 637-658 (1976).

17. J. E. Hansen, "Radiative transfer by doubling very thin layers," Astrophys. J. 155, 565-573 (1969).

18. L. G. Henyey and J. L. Greenstein, "Diffuse radiation in the galaxy," Astrophys. J. 93, 70-83 (1941).

19. G. Yoon, S. A. Prahl, and A. J. Welch, "Accuracies of the diffusion approximation and its similarity relations for laser irradiated biological media," Appl. Opt. 28, 2250-2255 (1989).

20. C.-C. Sun, T.-X. Lee, S.-H. Ma, Y.-L. Lee, and S.-M. Huang, "Precise optical modeling for LED lighting verified by cross correlation in the midfield region," Opt. Lett. 31, 2193-2195 (2006).

21. I. Moreno and C.-C. Sun, "Modeling the radiation pattern of LEDs," Opt. Express 16, 1808-1819 (2008). 